\title{
PROPER HOLOMORPHIC MAPPINGS
}

\author{
BY ERIC BEDFORD ${ }^{1}$
}

\section{Contents}

§1. Structure and Examples

§2. Analytic Projection Operator

§3. Boundary Regularity

§. Generic Branching

§. Factorization

§6. Mapping into Higher Dimensional Spaces

Introduction. Let us recall that a mapping $F: X \rightarrow Y$ is proper if $f^{-1}(K)$ is a compact subset of $X$ whenever $K \subset Y$ is compact. If $X$ and $Y$ are complex spaces, and if $F: X \rightarrow Y$ is a proper holomorphic mapping, then $\left.F^{-(} y_{0}\right)$ is a compact analytic subvariety of $X$ for all points $y_{0} \in Y$. Proper mappings between complex spaces were studied from the general point of view of complex spaces in the 1950s and early 60s (see Remmert-Stein [78]). Two results from this era are a factorization theorem of Stein [88] and the Remmert Proper Mapping theorem: If $f: X \rightarrow Y$ is a proper mapping, and if $S \subset X$ is a subvariety of $X$, then $f(S)$ is a subvariety of $Y$.

Here we consider a special case: proper mappings $F: \Omega \rightarrow D$ where $\Omega \subset \subset X$ $=\mathrm{C}^{n}$ and $D \subset \subset Y=\mathrm{C}^{N}$ are smoothly bounded domains. ${ }^{2}$ The letters $\Omega$ and $D$ will always denote domains of $\mathbf{C}^{n}$, and a "proper mapping" will always be assumed to be holomorphic. (In many cases the same results are valid in the case where $X$ and $Y$ are Stein manifolds, although we will not emphasize this point.)

It is evident that a mapping $F: \Omega \rightarrow D$ is proper if and only if $f$ maps $\partial \Omega$ to $\partial D$ in the following sense:

$$
\begin{aligned}
& \text { if }\left\{z_{j}\right\} \subset \Omega \text { is a sequence with } \underset{j \rightarrow \infty}{\lim \operatorname{dist}}\left(z_{j}, \partial \Omega\right)=0 \text {, then } \\
& \underset{j \rightarrow \infty}{\lim \operatorname{dist}}\left(f\left(z_{j}\right), \partial D\right)=0 .
\end{aligned}
$$

This is an expanded version of an hour address given on November 12, 1982 at the American Mathematical Society meeting in Baton Rouge, Louisiana; received by the editors September 9, 1983.

1980 Mathematics Subject Classification. Primary 32H99, 32E35; Secondary 32H10, 32F20, $32 \mathrm{~F} 15$

${ }^{1}$ Partially supported by the N.S. F.

${ }^{2}$ In this case proper mappings are also known as "finite mappings". 
Thus proper mappings $f: \Omega \rightarrow D$ lead naturally to the geometric function theory of mappings taking $\partial \Omega$ to $\partial D$.

The case where proper mappings are best understood is when the domains are strongly pseudoconvex. Pinčuk [73] has shown that a proper mapping $f: \Omega \rightarrow D$ between strongly pseudoconvex domains is locally biholomorphic. In fact, this result remains true if $D$ is only assumed to be (weakly) pseudoconvex (see [20, 38]). It has been shown, too, that if $\partial \Omega$ and $\partial D$ are strongly pseudoconvex and real analytic then a germ of a holomorphic mapping $f$ with $f(U \cap \partial \Omega) \subset \partial D$ for some open $U$ containing $z_{0} \in \partial \Omega$ may be analytically continued along $\partial \Omega$ (see $[\mathbf{7 1}, 72,27])$.

In this article we will survey some recent results on proper mappings between smoothly bounded domains. Biholomorphic mappings, of course, are a special case of proper ones. But here we want to study the features of proper maps that do not arise already in the locally biholomorphic case. Thus we focus our attention on the behavior of singular, i.e. branched, mappings, with emphasis on the smoothness and branching behavior at the boundary.

For boundary smoothness of proper mappings, we will present one of the proofs of S. Bell. We will not attempt to discuss or even mention the numerous other methods that have been developed; the reader may consult the references given in $[26,37,43]$. The literature on biholomorphic maps and automorphisms is so extensive that we cannot adequately present it here and, therefore, have omitted it almost entirely.

A related topic, not discussed here, is the proper mapping of polyhedra (see $[57,78,79,80,50,85])$. Another topic which we have omitted is the holomorphic correspondence, i.e. multiple-valued analytic mapping. This arises naturally in problems where it is desirable to study $f^{-1}$, for example, in factorization. The reader is referred to $[89,86,80,13,14,97]$.

Finally we note that, except in $\$ 6$, we assume $f$ is equidimensional, i.e. $\operatorname{dim} \Omega=\operatorname{dim} D$.

1. Structure and examples. First we give some examples of proper mappings, then we describe the basic structure of a proper map in terms of a branched cover, and we conclude this section with some more examples.

EXAMPLE (UNIT Disk). The proper mappings $f: \Delta \rightarrow \Delta$ are finite Blaschke products, i.e.

$$
f(z)=e^{i \phi} \prod_{j=1}^{k} \frac{z-\alpha_{j}}{1-\bar{\alpha}_{j} z},
$$

where $\alpha_{1}, \ldots, \alpha_{k} \in \Delta$, and $\phi \in \mathbf{R}$.

Proper mappings of the polydisk $\Delta^{n}=\Delta \times \cdots \times \Delta$ to itself are thus determined by the following theorem (see $[78,69,80])$.

THEOREM. If $\Omega_{1}, \ldots, \Omega_{n}, D_{1}, \ldots, D_{n} \subset \mathrm{C}$ are bounded domains, and if $f$ : $\Omega_{1} \times \cdots \times \Omega_{n} \rightarrow D_{1} \times \cdots \times D_{n}$ is a proper mapping, then there are a permutation $\sigma$ of $\{1, \ldots, n\}$ and proper maps $f_{j}: \Omega_{\sigma(j)} \rightarrow D_{j}$ such that

$$
f\left(z_{1}, \ldots, z_{n}\right)=\left(f_{1}\left(z_{\sigma(1)}\right), \ldots, f_{n}\left(z_{\sigma(n)}\right)\right) \text {. }
$$


EXAmple (The BALL). The proper self-maps of the unit ball $\mathbf{B}^{n}=\left\{z \in \mathbf{C}^{n}\right.$ : $\left.\left|z_{1}\right|^{2}+\cdots+\left|z_{n}\right|^{2}<1\right\}, n \geqslant 2$, were shown to be automorphisms by Alexander [3] (see also [71, 82]).

The existence of many proper mappings is given by a result of Grunsky [55] and Ahlfors [1].

THEOREM. If $M$ is a finite Riemann surface with nondegenerate boundary components, then there exists a proper mapping $f: M \rightarrow \Delta$.

In general, however, given two Riemann surfaces $M$ and $N$, it does not seem easy to say whether there exists a proper mapping $f: M \rightarrow N$.

Now we turn to the general structure of a proper mapping $f: \Omega \rightarrow D$, where $\operatorname{dim} \Omega=\operatorname{dim} D=n$. Two objects of interest are the Jacobian determinant

$$
J_{f}(z)=\operatorname{det}\left(\partial f_{i}(z) / \partial z_{j}\right)
$$

and the branch locus

$$
V_{f}=\left\{z \in \Omega: J_{f}(z)=0\right\} .
$$

Evidently, if $z_{0} \in \Omega \backslash V_{f}$, then $f$ is a local diffeomorphism in a neighborhood of $z_{0}$.

Some basic properties of a proper mapping $f: \Omega \rightarrow D$ are:

(A) $f^{-1}\left(w_{0}\right)$ is a compact subvariety of $\Omega$ and is thus finite.

(B) $f$ is an open mapping.

(C) $f$ is not locally one-to-one in any neighborhood of any $z_{0} \in V_{f}$.

(D) $f$ has rank $n$ on a dense open subset of $\Omega$,

(E) the set of critical values $f\left(V_{f}\right)$ is a complex subvariety of $D$.

For simple proofs of these facts, see Chapter 15.1 of Rudin [81].

It follows that $f: \Omega \backslash f^{-1} f\left(V_{f}\right) \rightarrow D \backslash f\left(V_{f}\right)$ is a proper, unbranched cover. If $D$ is connected, then there is an integer $m$ such that $f^{-1}(w)$ contains exactly $m$ points for each $w \in D \backslash f\left(V_{f}\right)$. In fact, more is true:

(F) $f^{-1}(w)$ contains $m$ points if $w \notin f\left(V_{f}\right)$, and $f^{-1}(w)$ contains less than $m$ points if $w \in f\left(V_{f}\right)$.

Branching. We note two cases in which the branching behavior determines whether or not a mapping is biholomorphic. It is not hard to see that if $f: \Omega \rightarrow D$ is an unbranched proper map, and if $D$ is simply connected, then $f$ is a biholomorphism.

Less obvious is a result of Pincuk [70]: if $f: \Omega \rightarrow \Omega$ is an unbranched proper self-map, and if $\Omega$ has reasonable boundary, then $f$ is a biholomorphism.

Fornaess [48] has shown that there cannot be branching even at the boundary in the smooth, biholomorphic case: if $f: \Omega \rightarrow D$ is a biholomorphic mapping between pseudoconvex domains with $C^{2}$ boundaries and if $f \in C^{2}(\bar{\Omega})$, then $J_{f} \neq 0$ on $\bar{\Omega}$ and $f^{-1} \cdot \in C^{2}(\bar{D})$.

EXAMPle (REINHARDT Domains). Let $\Omega, D$ be Reinhardt domains, i.e. invariant under $\left(z_{1}, \ldots, z_{n}\right) \rightarrow\left(e^{i \theta_{1}} z_{1}, \ldots, e^{i \theta_{n}} z_{n}\right)$ for all $\theta_{1}, \ldots, \theta_{n} \in \mathbf{R}$. If $\Omega_{1}$, $\Omega_{2} \subset \subset C^{n}$ are Reinhardt domains satisfying

(a) $z_{1} \cdots z_{n} \neq 0$ for $\left(z_{1}, \ldots, z_{n}\right) \in \bar{\Omega}_{j}$,

(b) $\left(z_{1}^{-1}, \ldots, z_{n}^{-1}\right) \in \Omega_{j}$ if $\left(z_{1}, \ldots, z_{n}\right) \in \Omega_{i}$ 
for $j=1,2$, then every proper, unbranched cover $f: \Omega_{1} \rightarrow \Omega_{2}$ is of the form $f=\left(f_{1}, \ldots, f_{n}\right)$ with

$$
f_{j}=c_{j} z_{1}^{z_{j}^{1}} \cdots z_{n}^{\mu_{j}^{n}} \text { and } \mu_{j}^{k} \in \mathbf{Z} .
$$

This is Theorem 1 of [9].

Further, if $\Omega \subset \subset \mathbf{C}^{n}$ satisfies (a), and if $f: \Omega \rightarrow \Omega$ is a proper self-map, then $f \in \operatorname{Aut}(\Omega)$, and, in particular, $f$ has the form (*). This is seen because $f$ : $\Omega \backslash f^{-1} f\left(V_{f}\right) \rightarrow \Omega \backslash f\left(V_{f}\right)$ is an unbranched, proper cover. Thus for every $\sigma \in \pi_{1}\left(\Omega \backslash f\left(V_{f}\right)\right)$, there exists $\gamma \in \pi_{1}\left(\Omega \backslash f^{-1} f\left(V_{f}\right)\right)$ such that $f_{*} \gamma$ is an integer multiple of $\sigma$. Let $T$ be the $n \times n$ matrix with integer coefficients which represents $f_{*}: H_{1}(\Omega, Z) \rightarrow H_{1}(\Omega, Z)$ with respect to some basis. By the behavior of $f_{*}$ on the fundamental group, it follows that $\operatorname{det}(T) \neq 0$. Our conclusion now follows from Theorem 2 of [9].

For a rather general class of bounded Reinhardt domains $\Omega, D$, Barrett [7] has shown that a proper map $f: \Omega \rightarrow D$ extends holomorphically to a neighborhood of $\bar{\Omega}$. (See also $[64,65]$.)

Example (Complete, Circled Domains). If $\Omega, D$ are circled and complete (i.e. invariant under $\left.\left(z_{1}, \ldots, z_{n}\right) \rightarrow\left(\lambda z_{1}, \ldots, \lambda z_{n}\right), \lambda \in \mathbf{C},|\lambda| \leqslant 1\right)$, then every proper map $f: \Omega \rightarrow D$ with $f^{-1}(0)=\{0\}$ is polynomial (Bell [21]).

2. The analytic projection operator. The Bergman metric and kernel, while useful tools for biholomorphic mappings, are not invariant under proper mappings. The analytic (Bergman) projection operator, however, is invariant. This will be used in $\S 3$, where we describe the proof that proper mappings extend smoothly to the boundary.

If $\omega=h(z) d z_{1} \wedge \cdots \wedge d z_{n}$ and $\eta=k(z) d z_{1} \wedge \cdots \wedge d z_{n}$ are $(n, 0)$-forms on $\Omega$, then we may consider the inner product

$$
(\omega, \eta)_{\Omega}=i^{n^{2}} \int_{\Omega} \omega \wedge \bar{\eta},
$$

which is well defined (as integration of a $2 n$-form over a $2 n$-manifold) independently of any metric on $\Omega$. It is easily seen that

$$
\|\omega\|_{\Omega}^{2}=(\omega, \omega)_{\Omega}=2^{n} \int_{\Omega}|h|^{2} d V
$$

where $d V$ denotes the Euclidean volume. Let $L^{2}(\Omega)_{n, 0}=\{(n, 0)$-forms $\omega$ with $\left.\|\omega\|^{2}<\infty\right)$, and let $L_{a}^{2}(\Omega)_{n, 0}$ denote the holomorphic $n$-forms in $L^{2}(\Omega)_{n, 0}$. Thus $L_{a}^{2}(\Omega)_{n, 0}$ is a closed subspace of $L^{2}(\Omega)_{n, 0}$, and we may define the orthogonal projection

$$
P_{\Omega}: L^{2}(\Omega)_{n, 0} \rightarrow L_{a}^{2}(\Omega)_{n, 0} .
$$

We recall that if $f: \Omega \rightarrow D$ is a holomorphic mapping, then the pull-back operator $f^{*}$ is defined by

$$
f^{*}\left(h(w) d w_{1} \wedge \cdots \wedge d w_{n}\right)=h(f(z)) J_{f}(z) d z_{1} \wedge \cdots \wedge d z_{n} .
$$

Thus, if $f: \Omega \rightarrow D$ is a $p$-to-1 proper mapping, then by the usual "change of variables" formula, we have

$$
\int_{\Omega}|h(f(z))|^{2}\left|J_{f}(z)\right|^{2} D V_{z}=p \int_{D}|h(w)|^{2} D V_{w},
$$


since $\left|J_{f}\right|^{2}$ is the same as the real Jacobian of $f$. It follows, then, that for $\eta \in L^{2}(\Omega)$,

$$
\left\|f^{*} \eta\right\|_{\Omega}^{2}=p\|\eta\|_{D}^{2}
$$

and thus $f^{*}: L^{2}(D)_{n, 0} \rightarrow L^{2}(\Omega)_{n, 0}$.

The relation between the holomorphic projection $P$ and proper mappings was given by Bell [19].

LEMMA. If $f: \Omega \rightarrow D$ is proper, then $P_{\Omega} f^{*}=f^{*} P_{D}$.

Proof. There are two cases to consider.

Case (i). If $\eta \in L_{a}^{2}(D)$, then $P_{D} \eta=\eta$. On the other hand, by (2.1), $f^{*} \eta \in$ $L_{a}^{2}(\Omega)$, so $P_{\Omega} f^{*} \eta=f^{*} \eta$.

Case (ii). If $\eta \perp L_{a}^{2}(D)_{n, 0}$, we must show $f^{*} \eta \perp L_{a}^{2}(\Omega)_{n, 0}$. Let us first assume that $\eta=\left(\partial / \partial w_{j}\right) \phi(w) d w_{1} \wedge \cdots \wedge d w_{n}$, where $\phi \in C_{0}^{\infty}\left(D \backslash f\left(V_{f}\right)\right)$. If $g \in L_{a}^{2}(\Omega)_{n, 0}$ then, setting $V=f^{-1} f\left(V_{f}\right)$, and denoting the local inverses of $f$ by $F_{1}, \ldots, F_{q}$ we have

$$
\begin{aligned}
\int_{\Omega \backslash V} & f^{*} \eta \wedge \bar{\omega} \\
& =\int_{\Omega \backslash V} \frac{\partial}{\partial w_{j}} \phi(f(z)) J_{f}(z) \wedge \overline{g(z)} D z_{1} \wedge \cdots \wedge d z_{n} \wedge d \bar{z}_{1} \wedge \cdots \wedge d \bar{z}_{n} \\
& =\sum_{k=1}^{q} \int_{D \backslash f\left(V_{f}\right)} \frac{\partial}{\partial w_{j}} \phi(w) \overline{g\left(F_{k}(w)\right)} \overline{J_{F_{k}}(w)} d w_{1} \wedge \cdots \wedge d w_{n} \\
\quad= & \sum_{k=1}^{q} \int_{D \backslash f\left(V_{f}\right)} \phi(w) \frac{\partial}{\partial w_{j}} \overline{\left(g\left(F_{k}(w)\right) J_{F_{k}}(w)\right)} d w \wedge d \bar{w}=0 .
\end{aligned}
$$

We conclude that $f^{*} \eta \perp L_{a}^{2}(\Omega)_{n, 0}$.

Finally, the $(n, 0)$-forms

$$
\eta=\sum_{j=1}^{n} \frac{\partial \phi_{j}}{\partial w_{j}} d w_{1} \wedge \cdots \wedge d w_{n}
$$

$\phi_{j} \in C_{0}^{\infty}\left(D \backslash f\left(V_{f}\right)\right)$, are dense in $L_{a}^{2}(D)_{n, 0}^{\perp}$. For if $h \in L^{2}(D)_{n, 0}^{\perp}$ and $(h, \eta)_{D}$ $=0$, integration by parts shows that $h$ is holomorphic on $D \backslash V$. By the Riemann Removable Singularity Theorem, $h \in L_{a}^{2}(D)_{n, 0}$, and thus $h=0$.

REMARK. A similar argument was given by Pinčuk [74] to show that the kernel functions themselves satisfy

$$
K_{\Omega}(z) \geqslant(1 / p)\left|J_{f}(z)\right|^{2} K_{D}(f(z)) .
$$

Condition $R$. In the context of boundary regularity it is important to know whether the holomorphic projection preserves smooth functions, i.e.

$$
P_{\Omega}\left(C_{n, 0}^{\infty}(\bar{\Omega})\right) \subset C_{n, 0}^{\infty}(\bar{\Omega}) .
$$

Domains with regular projection, i.e. which satisfy (2.2), are said to satisfy Condition $R$. 
One approach to Condition $R$ is given through the $\bar{\partial}$-Neumann problem. The $\bar{\partial}$-solving operator $S$ corresponding to the $\bar{\partial}$-Neumann problem is given by the map (if it exists)

$$
S:\left\{\mu \in L^{2}(\Omega)_{0,1}: \bar{\partial} \mu=0\right\} \rightarrow L^{2}(\Omega)
$$

such that $S(\mu)$ satisfies

$$
\bar{\partial} S(\mu)=\mu
$$

and $S(\mu)$ minimizes $\int_{\Omega}|S(\mu)|^{2} d V$ over all solutions of (*). If we identify $(n, 0)$-forms with functions in the obvious way, then it is evident that

$$
f-P_{\Omega} f=S(\bar{\partial} f) .
$$

Thus $\Omega$ will satisfy Condition $R$ if

$$
S:\left\{\mu \in C^{\infty}(\bar{\Omega})_{0,1}: \bar{\partial} \mu=0\right\} \rightarrow C^{\infty}(\bar{\Omega}) .
$$

So far, the greatest source of domains known to satisfy Condition $\mathbf{R}$ is from condition (2.3), which is obtained from subelliptic estimates for the $\bar{\partial}$-Neumann operator. The Neumann operator $N$ is an operator which inverts the Laplacian $\square=\sum \partial^{2} / \partial z_{j} \partial \bar{z}_{j}$ subject to the " $\bar{\partial}-$ Neumann" boundary conditions. That is, for a $(0,1)$-form $\mu$ on $\Omega, N \mu$ is a $(0,1)$-form solving $\square N \mu=\mu$ on $\Omega$ and satisfying the $\bar{\partial}-$ Neumann conditions

$$
N \mu\lrcorner \bar{\partial} r=0, \quad \bar{\partial}(N \mu)\lrcorner \bar{\partial} r=0
$$

on $\partial \Omega$. These boundary conditions are degenerate (noncoercive), but they serve as a substitute for the orthogonality condition corresponding to $(* *)$. Finally, if the Neumann operator exists, there is a Hodge-type decomposition for $N$, from which we have $S \mu=\bar{\partial} * N \mu$ for $\mu$ satisfying $\bar{\partial} \mu=0$ (see the survey article of Kohn [60]).

The work of Kohn [59] showed that (2.3) holds under certain geometric hypotheses on $\partial \Omega$. Diederich and Fornaess [36] have proven, in particular, that these hypotheses are satisfied for pseudoconvex domains with real-analytic boundaries. D. Catlin [32] has shown, more generally, that (2.3) holds even for pseudoconvex domains which have finite type in the sense of $\mathrm{D}^{\prime}$ Angelo [95].

Although pseudoconvexity (or at least 1-convexity) is a necessary condition for (2.3), it is not clear what role pseudoconvexity plays in Condition $R$. Bell-Boas [25], Bell [23] and Barrett [5] have shown that (2.2) holds in cases where (2.3) does not hold, i.e. where $\Omega$ is not pseudoconvex and $\bar{\partial}$ is not solvable. On the other hand, Barrett [8] has recently found a smoothly bounded but not pseudoconvex domain for which Condition $\mathrm{R}$ fails.

3. Boundary regularity. The following conjecture is well known but unresolved: If $\Omega, D \subset \subset \mathrm{C}^{n}$ are domains with smooth boundary, then every proper mapping $f: \Omega \rightarrow D$ extends smoothly to $\bar{\Omega}$.

Before discussing some partial results in this direction, we give some examples of poorly behaved proper mappings.

Let $\Omega=\left\{(z, w) \in \mathbf{C}^{2}:|z|<1\right\}, \mathbf{T}=\mathbf{C} /(\mathbf{Z}+i \mathbf{Z})$, and $\Delta=\{z \in \mathbf{C}:|z|<1\}$.

EXAMPLE. If $f(z, w): \Omega \rightarrow \Omega$ is given by $f(z, w)=(z, w+g(z))$, where $g(z)$ is any analytic function on $\Delta$, then $f$ is proper. 
EXAMPLE. The function $f: \Delta \times \mathbf{T} \rightarrow \Delta \times \mathbf{T}$ given by $f(z, w)=(z, w+g(z))$ (as above) is proper.

EXAMPLE. The function $G(z, w)=\left(z,(w-g(z))^{2}\right)$ yields a proper mapping $G: \Omega \rightarrow \Omega$ and a proper mapping $G: \Delta \times \mathbf{T} \rightarrow \Delta \times \mathbf{T}$.

In the first two examples, $f$ does not extend smoothly to the boundary if $g$ does not extend smoothly to $\bar{\Delta}$. In the third example, $g$ may be chosen so that $V_{G}=\{w=g(z): z \in \Delta\}$ is dense in $\partial \Omega$ (or $\partial \Delta \times \mathbf{T}$ ).

EXAMPle (PIECEWISE - SMOOTH BoundaRY). Fridman [52] has shown that there is a domain $\Omega \subset \subset C^{2}$ with piecewise-smooth boundary, and there is a biholomorphism $f: \Omega \rightarrow \Delta^{2}$ which does not extend continuously to $\bar{\Omega}$.

The first step to establishing boundary regularity is to show that boundary distances are preserved. For this we use a result of Diederich and Fornaess [35]: if $D \subset \subset C^{n}$ is a pseudoconvex domain with $C^{2}$ boundary, there exists $r \in C^{2}(\bar{D})$ with $\{-r<0\}=D,\{r=0\}=\partial D, \nabla r \neq 0$ on $\partial D$, and $-(-r)^{\varepsilon}$ is plurisubharmonic on $D$ for some $\varepsilon>0$. The following argument is due to Henkin and Pinčuk [57, 70, 76].

LEMMA 1. If $\Omega, D \subset \subset C^{n}$ are pseudoconvex with $C^{2}$ boundary, and if $f: \Omega \rightarrow D$ is a proper mapping, then there exists $\varepsilon>0$ such that

$$
\varepsilon(\operatorname{dist}(f(z), \partial D))^{1 / \varepsilon} \leqslant \operatorname{dist}(z, \partial \Omega) \leqslant \varepsilon^{-1}(\operatorname{dist}(f(z), \partial D))^{\varepsilon} .
$$

Proof. Let $r$ be the smooth defining function above. Then $|r(w)|$ is estimated above and below by $C \operatorname{dist}(w, \partial D)$. Since $f$ is proper, $-(-r(f(z)))^{\varepsilon}$ is a psh. exhaustion function for $\Omega$. By the Hopf Lemma, there is a constant $C>0$ such that

$$
-(-r(f(z)))^{\varepsilon} \leqslant-C \operatorname{dist}(z, \partial \Omega)
$$

It follows, then, that

$$
\operatorname{dist}(f(z), \partial D)^{\varepsilon} \geqslant C \operatorname{dist}(z, \partial \Omega) \text {. }
$$

The other inequality is obtained in the same manner because if $\rho$ is a psh. exhaustion for $\Omega$, then

$$
\psi(w)=\max _{z \in f^{-1}(w)} \rho(z)
$$

is a psh. exhaustion for $D$.

Now since $f(z)=\left(f_{1}(z), \ldots, f_{n}(z)\right)$ is bounded, the Cauchy estimates give

$$
\left|D^{\alpha} f_{j}(z)\right| \leqslant M \operatorname{dist}(z, \partial \Omega)^{-|\alpha|} \text {. }
$$

Thus from Lemma 1 and the Cauchy estimates, we obtain

LEMMA 2. $f^{*}\left(C_{0}^{\infty}(\bar{D})_{n, 0}\right) \subset C_{0}^{\infty}(\bar{\Omega})_{n, 0}$.

We will also need the following.

LEMMA 3. $P\left(C_{0}^{\infty}(\bar{D})_{n, 0}\right)=P\left(C^{\infty}(\bar{D})_{n, 0}\right)$.

Proof. For $\phi \in C^{\infty}(\bar{D})$, we want to find $\psi \in C_{0}^{\infty}(\bar{D})$ such that $P \phi=P \psi$. We let $r \in C^{\infty}(\bar{D})$ be a defining function for $D$. By a partition of unity, we 
may assume that the support of $\phi$ is small enough that $\partial r / \partial z_{1} \neq 0$ on $\partial \Omega \cap \operatorname{supp} \phi$.

We construct $\psi$ of the form

$$
\psi=\phi-\frac{\partial}{\partial z_{1}}\left(\sum_{j=1}^{\infty} \chi_{j} r^{j}\right) .
$$

We may arrange to have $\psi \in C_{0}^{\infty}(\bar{D})$ since we may solve successively for $\chi_{j}$. This starts as

$$
\chi_{1} \frac{\partial r}{\partial z_{1}}=\phi \quad(\bmod O(r))
$$

and proceeds inductively as

$$
\phi-\frac{\partial}{\partial z_{1}}\left(\sum_{j=1}^{k-1} \chi_{j} r^{j}\right)=k r^{k-1} \chi_{k}\left(\bmod O\left(r^{k}\right)\right) .
$$

This determines the value of $\left.\chi_{j}\right|_{\partial D}$ in terms of $\chi_{1}, \ldots, \chi_{j-1}$ and their derivatives on $\partial D$. Clearly $\chi_{j} \in C^{\infty}(\bar{D})$ may be chosen so that the summation converges in $C^{\infty}(\bar{D})$.

Finally, it is evident that $P_{D} \phi=P_{D} \psi$ since $\partial h / \partial z_{1} \perp L_{a}^{2}(D)$ for all $h \in C^{1}(\bar{D})$ with $h=0$ on $\partial D$.

THEOREM. Let $\Omega, D \subset \subset \mathbf{C}^{n}$ be smoothly bounded and pseudoconvex, and let $\Omega$ satisfy Condition $\mathrm{R}$. Then if $f: \Omega \rightarrow D$ is proper, $J_{f} f_{1}^{\alpha_{1}} \cdots f_{n}^{\alpha_{n}} \in C^{\infty}(\bar{\Omega})$ for all integers $\alpha_{1}, \ldots, \alpha_{n} \geqslant 0$.

PRoof. We set $\eta=w_{1}^{\alpha_{1}} \cdots w_{n}^{\alpha_{n}} d w_{1} \wedge \cdots \wedge d w_{n}$. By the preceding lemma, there is a smooth form $\xi \in C_{0}^{\infty}(\bar{D})_{n, 0}$ with $P_{D} \xi=\eta$. Further,

$$
f^{*} \eta=f_{1}^{\alpha_{1}} \cdots f_{n}^{\alpha_{n}} J_{f} d z_{1} \wedge \cdots \wedge d z_{n} .
$$

But

$$
f^{*} \eta=f^{*} P_{D} \xi=P_{\Omega} f^{*} \xi \in C^{\infty}(\bar{\Omega})_{n, 0}
$$

since $f^{*} \xi$ is a smooth form on $\bar{\Omega}$, and $\Omega$ satisfies Condition R. This completes the proof.

This theorem shows that $f(z)$ is smooth on a dense subset of the boundary, i.e. on $\left\{z \in \partial \Omega: J_{f}(z) \neq 0\right\}$. This is already a useful result: for instance, it follows from Webster [91] that if $\partial \Omega$ and $\partial D$ are, in addition, defined by polynomial equations, then $f$ is algebraic.

By the theorem above, the problem of showing that $f \in C^{\infty}(\bar{\Omega})$ is thus reduced to a problem of division, which was solved by Bell and Catlin [26] and Diederich and Fornaess [39]. They prove the finite vanishing of $J_{f}$ at $\partial \Omega$ and then a Division Theorem, both of which are of independent interest and are stated here.

LeMma (Finite VANishing of THE JACOBIAN). If $f: \Omega \rightarrow D$ is proper, and if $\Omega, D \subset \subset C^{n}$ are smoothly bounded and pseudoconvex, then $J_{f}$ vanishes to only finite order at $\partial \Omega$, i.e. there is no $z_{0} \in \partial \Omega$ such that $\left|J_{f}(z)\right|=O\left(\left|z-z_{0}\right|^{m}\right)$ for $m=1,2,3, \ldots$. 
Division TheOREM. Let $\Omega \subset \subset \mathbf{C}^{n}$ be a domain with smooth boundary. If $u(z)$ and $f(z)$ are holomorphic on $\Omega$, if $u \in C^{\infty}(\bar{\Omega})$ vanishes to finite order at $\partial \Omega$, and if $u f^{j} \in C^{\infty}(\bar{\Omega})$ for $j=1,2,3, \ldots$, then $f \in C^{\infty}(\bar{\Omega})$.

From these results it follows that the conjecture stated at the beginning of this section is valid for pseudoconvex $\Omega$ satisfying Condition $R$. In a subsequent note [16], it was shown that these arguments apply also to domains $\Omega, D$ inside a Stein manifold. It has also been shown that the pseudoconvexity hypothesis may be dropped if both $\Omega$ and $D$ satisfy Condition $\mathrm{R}$ (see Bell [18]).

These methods also yield extensions to a neighborhood of $\bar{\Omega}$ in some cases $($ see $[20,6])$.

4. Generic branching. If $f: \Omega \rightarrow D$ is proper, then so is

$$
f_{0}=\left.f\right|_{V}: V \rightarrow W,
$$

where $V=V_{f}$ and $W=f\left(V_{f}\right)$. Certain kinds of local singular behavior are not possible for proper mappings. For instance, a proper mapping cannot look like $h(z, w)=(z, z w)$ near the origin, since $h^{-1}(0,0)=\{0\} \times \mathbf{C}$ has positive dimension. It does not seem to be known, however, just what varieties $V, W$ and proper mappings $f_{0}: V \rightarrow W$ can arise as in (4.1) (cf. also the conjecture in [87]).

Although the exact branching behavior is not known (except in the case $\Omega=\mathbf{B}^{n}$, in $\$ 5$ ), the generic branching behavior is easy to describe, and there is a relation between the generic branching order of a mapping at the boundary and the order of vanishing of the Levi form. The following is an elementary illustration of this.

EXAMPLE. If $f(z, w)=\left(z, w^{2}\right)$, then $f: \Omega_{1} \rightarrow \Omega_{2}$ and $f: \Omega_{2} \rightarrow \Omega_{3}$ are proper, where

$$
\begin{aligned}
& \Omega_{1}=\left\{|z|^{2}+|w|^{4}<1\right\}, \\
& \Omega_{2}=\left\{|z|^{2}+|w|^{2}<1\right\}, \\
& \Omega_{3}=\left\{|z|^{2}+|w|<1\right\} .
\end{aligned}
$$

The branch locus in both cases is $V_{f}=\Delta \times\{0\}$. Note that $\Omega_{1}$ is Levi flat at $\partial \Omega_{1} \cap \bar{V}_{f}, \Omega_{2}$ is strongly pseudoconvex at $\partial \Omega_{2} \cap \bar{V}_{f}$, and $\Omega_{3}$ is not smooth at $\partial \Omega_{3} \cap \bar{V}_{f}$.

The point is that the branching order decreases the degree of Levi flatness. If the boundary is nowhere Levi flat (i.e. strongly pseudoconvex), then a branched mapping has a nonsmooth image.

We note that the nature of the nonsmooth image can never be as "nice" as piecewise-smooth according to the following result of Pinčuk [74].

Theorem. Let $D, G \subset \mathbf{C}^{n}(n>1)$ be bounded, pseudoconvex domains, $G$ having boundary of class $C^{2}$, and $D$ having piecewise $C^{2}$-smooth, but not smooth, boundary. Then there does not exist a proper mapping $g: G \rightarrow D$.

Generic branch points. Given a point $z_{0} \in V_{f}$, we may move it slightly so that $z_{0}$ is a regular point of $V_{f}$, the rank of $f_{0}$ is $n-1$ at $z_{0}$, and $f\left(V_{f}\right)$ is regular at 
$f\left(z_{0}\right)$. Thus we may make holomorphic changes of coordinates in neighborhoods of $z_{0}$ and $f\left(z_{0}\right)$ so that $z_{0}=f\left(z_{0}\right)=0, V_{f}=\left\{z_{n}=0\right\}$ near $z_{0}=0$, $f\left(V_{f}\right)=\left\{z_{n}=0\right\}$ near $f\left(z_{0}\right)=0$, and $f_{0}\left(z_{1}, \ldots, z_{n-1}\right)=\left(z_{1}, \ldots, z_{n-1}\right)$ holds for $z \in V_{f}$ near $z_{0}=0$.

Since $\left\{f_{n}(z)=0\right\}=\left\{z_{n}=0\right\}$ holds in a neighborhood of $z_{0}$, we have

$$
f_{n}(z)=\sum_{j=1}^{\infty} a_{j}\left(z^{\prime}\right) z_{n}^{j}
$$

where $a_{j}$ is analytic in $z^{\prime}=\left(z_{1}, \ldots, z_{n-1}\right)$. Moving $z_{0}$ slightly within $V_{f}$, we may assume

$$
f_{n}(z)=\sum_{j=M}^{\infty} a_{j}\left(z^{\prime}\right) z_{n}^{j}
$$

and $a_{M}(0) \neq 0$. Thus

$$
f_{n}(z)=z_{n}^{M} U(z)
$$

where $U(0) \neq 0$. By a change of coordinates in $D, z_{n}^{\prime}=z_{n}(U(z))^{1 / M}$, we may assume $f_{n}(z)=z_{n}^{M}$ in a neighborhood of $z_{0}$.

We conclude this discussion with the observation that for a generic point $z_{0} \in V_{f}$, holomorphic changes of coordinates may be made at $z_{0}$ and $f\left(z_{0}\right)$ so that

$$
f(z)=\left(z_{1}+z_{n} g_{1}(z), \ldots, z_{n-1}+z_{n} g_{n-1}(z), z_{n}^{M}\right) .
$$

The branching order of $f$ at $z_{0}$ is $\operatorname{Ord}\left(f, z_{0}\right)=M-1$, and is constant on the irreducible component of $V_{f}$ containing $z_{0}$.

REMARK 1. By a change of coordinates of the form

$$
z_{j}^{\prime}=z_{j}-g_{j}(0) z_{n}-\frac{\partial g_{j}}{\partial z_{n}}(0) z_{n}^{2}, \quad 1 \leqslant j \leqslant n-1, z_{n}^{\prime}=z_{n},
$$

we may assume (4.2) satisfies $g_{j}(0)=\partial g_{j}(0) / \partial z_{n}=0$.

REMARK 2. If $\partial \Omega$ is strongly pseudoconvex and $f: \Omega \rightarrow D, f \in C^{\infty}(\bar{\Omega})$, is proper, then we can choose a generic $z_{0} \in \bar{V}_{f} \cap \partial \Omega$ such that (4.2) holds to arbitrarily high order.

Next we give a measure of flatness of the boundary. If $r \in C^{\infty}(\bar{D}), D=$ $\{r<0\}$ and $\nabla r \neq 0$ on $\partial D$, then the determinant of the Levi form is given by

$$
\lambda_{D}(w)=-\operatorname{det}\left(\begin{array}{c|c}
0 & \partial r / \partial \bar{w}_{j} \\
\hline \partial r / \partial w_{i} & \partial^{2} r / \partial w_{i} \partial \bar{w}_{j}
\end{array}\right)|\nabla r|^{-n-1} .
$$

A point $w_{0} \in \partial D$ is strongly pseudoconvex if the Levi form is positive definite and thus $\lambda_{D}\left(w_{0}\right)>0$. If $f: \Omega \rightarrow D$ is proper, then $\rho=r \circ f$ is a defining function for $\Omega$, and by the chain rule we may compute

$$
\lambda_{\Omega}(z)=\lambda_{D}(f(z))\left|J_{f}(z)\right|^{2}
$$

for $z \in \partial \Omega$. [The fact that $\rho$ is a defining function, i.e. $\nabla \rho \neq 0$ on $\partial \Omega$, is seen, since by [35] we may take $r$ to be a smooth defining function such that $-(-r)^{2 / 3}$ is psh. on $D$ near $f(z)$ and then apply the Hopf Lemma to $-(-\rho)^{2 / 3}$.] 
To illustrate the utility of (4.3) we give a result which was proved in various forms in $[29,73,20,38]$ for conclusion (i) and $[74,18]$ for conclusion (ii).

THEOREM. If $\Omega, D \subset \subset \mathrm{C}^{n}$ are smoothly bounded pseudoconvex domains and $\Omega$ is strongly pseudoconvex, then for any proper mapping $f: \Omega \rightarrow D$ it follows that

(i) $f$ is a local biholomorphism;

(ii) $D$ is strongly pseudoconvex.

Proof. By $\S 1$, if $f$ is not a local biholomorphism at some point of $\Omega$, then $V_{f} \neq \varnothing$, and thus $\bar{V}_{f} \cap \partial \Omega \neq \varnothing$. By $\S 3, J_{f} \in C^{\infty}(\bar{\Omega})$ and thus $J_{f}\left(z_{0}\right)=0$ for $z_{0} \in \bar{V}_{f} \cap \partial \Omega$. By (4.3) it follows that $\lambda_{\Omega}\left(z_{0}\right)=0$ and thus $\Omega$ is Levi flat at $z_{0}$, which is a contradiction.

It also follows from (4.3) that $\lambda_{D} \neq 0$ at $f\left(z_{0}\right)$, and thus $D$ is strongly pseudoconvex at all points $f(\partial \Omega) \subset \partial D$. Since $f \in C(\bar{\Omega})$ and $f$ is proper, $f(\partial \Omega)=\partial D$, which proves (ii).

From (4.3) it is evident that $\lambda$ is not a biholomorphic invariant of the boundary, although the condition $\lambda=0$ is invariant. If $z_{0} \in \partial \Omega$, then we may define

$$
\begin{aligned}
& \tau\left(z_{0}\right)=\text { order of vanishing of } \lambda(z) \text { at } z=z_{0} \\
& =\min \{m: \text { for every tangential differential operator } \\
& \left.\qquad P \text { on } \partial \Omega \text { of order } m, P\left(\lambda\left(z_{0}\right)\right)=0\right\},
\end{aligned}
$$

and $\tau$ is a biholomorphic invariant. A more thorough discussion of the invariants of the Levi form is given in [95]. From (4.2) and (4.3) we obtain a relation between the generic branching order and $\tau$.

PROPOSITION. If $f: \Omega \rightarrow D$ is a proper mapping, $f \in C^{\infty}(\bar{\Omega})$, and $z_{0} \in \partial \Omega$ is a generic branch point, then $\tau\left(z_{0}\right)+2=\left(\operatorname{Ord}\left(f, z_{0}\right)+1\right)\left(\tau\left(f\left(z_{0}\right)\right)+2\right)$.

If $\partial \Omega$ is real-analytic, we may make a semianalytic stratification of the set $\{z \in \partial \Omega: \lambda(z)=0\}$ into sets where $\tau$ is constant. From this we may extract a finite number of connected, real-analytic manifolds $\Gamma^{1}, \ldots, \Gamma^{k}$ with the properties:

(1) $\tau=\tau_{j}$ is constant on $\Gamma^{j}, 1 \leqslant j \leqslant k$;

(2) there is a connected complex $(n-1)$-manifold $\tilde{\Gamma}^{j} \subset \mathbf{C}^{n}$ with $\Gamma^{j} \subset \tilde{\Gamma}^{j}$, $1 \leqslant j \leqslant k$.

The details of this construction are given in [11]. The theorem below shows that if $\Omega$ has real-analytic, pseudoconvex boundary, then there are only finitely many possibilities for $V_{f}$, which are determined by $\partial \Omega$. Further, for a proper mapping $f: \Omega \rightarrow D$ between two domains with real-analytic boundaries, the special manifolds in $\partial \Omega$ are taken onto the special manifolds of $\partial D$.

THEOREM. Let $\Omega \subset \subset \mathbf{C}^{n}$ be a pseudoconvex domain with smooth, real-analytic boundary. Let $D \subset \subset \mathbf{C}^{n}$ be a domain with smooth boundary, and let $f: \Omega \rightarrow D$ be a proper mapping. Then for each irreducible component $V$ of $V_{f}$ there exists a point $z_{0} \in \Gamma^{j}$ and an open set $U$ containing $z_{0}$ such that $V \cap U=\tilde{\Gamma}^{j} \cap U$. Further, if $\partial D$ is real analytic, and $\Gamma^{\prime}$ is an element of the stratification of $\partial D$, then there exist $\Gamma^{i_{1}}, \ldots, \Gamma^{i_{r}} \subset \partial \Omega$ such that $\Gamma^{i_{1}} \cup \cdots \cup \Gamma^{i_{r}}$ is dense in $f^{-1}\left(\Gamma^{\prime}\right)$. 
Two applications of this theorem are given below. Since the corresponding values of $\tau$ must be related as in the above proposition, we have the following application to a specific case, which was also treated by Landucci [61].

THEOREM. If $\Omega=\left\{|z|^{2}+|w|^{2 p}<1\right\}$ and $D=\left\{|z|^{2}+|w|^{2 q}<1\right\}$, then there exists a proper mapping $f: \Omega \rightarrow D$ if and only if $q$ divides $p$.

If the theorem is applied to the iteration of a self-mapping, we obtain the following, which was proved in $[11,12,24]$.

THEOREM. If $\Omega \subset \subset \mathbf{C}^{n}$ is a pseudoconvex domain with smooth, real-analytic boundary, then every proper self-mapping $f: \Omega \rightarrow \Omega$ is an automorphism.

5. Factorization. Let $f: \Omega \rightarrow D$ be a proper mapping. We will discuss the existence of a subgroup $\Gamma_{f} \subset \operatorname{Aut}(\Omega)$ of the group of automorphisms with the properties:

(i) $f g(z)=f(z)$ for all $z \in \Omega$,

(ii) $f^{-1} f(z)=\cup_{g \in \Gamma_{f}} g(z)$ for all $z \in \Omega$.

The existence of the group $\Gamma_{f}$ gives a canonical factorization $f=\tilde{f} \eta$, where $\eta$ : $\Omega \rightarrow \Omega / \Gamma_{f}$ is the quotient mapping, and $\tilde{f}: \Omega / \Gamma_{f} \rightarrow D$ is a biholomorphism.

A factorization does not always exist. For example, let $\Omega=D=\Delta$ be the unit disk in $\mathbf{C}$, and let $f: \Delta \rightarrow \Delta$ be given by

$$
f(z)=\left(\frac{z-\alpha}{1-\bar{\alpha} z}\right)^{2}\left(\frac{z-\beta}{1-\bar{\beta} z}\right)
$$

where $\alpha, \beta \in \Delta, \alpha \neq \beta$. Note that $f^{-1}(0)=\{\alpha, \beta\}$, but that $f$ has different branching orders at $\alpha$ and $\beta$. Thus there cannot exist $g \in \operatorname{Aut}(\Delta)$ satisfying (i) above and $g(\alpha)=\beta$.

The existence of a factorization of a proper map $f: \Omega \rightarrow D$ is easily seen to be equivalent to the unbranched covering $f: \Omega \backslash f^{-1} f\left(V_{f}\right) \rightarrow D \backslash f\left(V_{f}\right)$ being normal. The reason for this is that $\Gamma_{f}$ are covering transformations by (i), and by (ii) the covering transformations are transitive.

If $f: \Omega \rightarrow D$ is generically $p$-to- 1 , and if $f$ can be factored, then it is evident that the order of the group $\Gamma_{f}$ is $p$. From $\S 1$,

$$
V_{f}=\left\{z \in \Omega: \text { the number of elements of } f^{-1} f(z) \text { is }<p\right\} .
$$

It follows that if $f$ can be factored, then

$$
V_{f}=\bigcup_{\substack{q \in \Gamma_{f} \\ g \neq \text { id }}} \operatorname{Fix}(q),
$$

where $\operatorname{Fix}(g)=\{z \in \Omega: q(z)=z\}$ is the set of fixed points of $g$.

It is useful to know that in certain cases factorizations do exist.

TheOREM (FACTORIZATION). Let $\Omega \subset \subset \mathbf{C}^{n}, n \geqslant 2$, be a simply connected, strongly pseudoconvex domain with $C^{\infty}$ smooth boundary. If $f: \Omega \rightarrow D$ is proper, then $f$ can be factored, i.e. there exists a subgroup $\Gamma_{f} \subset \operatorname{Aut}(\Omega)$ satisfying (i) and (ii). 
Proofs of this are given in $[13,15]$. Since those proofs use holomorphic correspondences, which we have not discussed here, let us give a sketch of the proof in [10], which assumes $f \in C^{\infty}(\bar{\Omega})$. We take $z_{0} \in \Omega \backslash f^{-1} f\left(V_{f}\right)$ and $z_{1} \in f^{-1} f\left(z_{0}\right)$ and consider the germ of a covering transformation taking $z_{0}$ to $z_{1}$. It must be shown that this transformation, which is a branch of $f^{-1} f$, may be analytically continued over $f^{-1} f\left(V_{f}\right)$. The problem reduces itself to showing that if $p_{1}, p_{2} \in f^{-1} f\left(V_{f}\right)$ are generic branch points, then $\operatorname{Ord}\left(f, p_{1}\right)=$ $\operatorname{Ord}\left(f, p_{2}\right)$. To show this we let $\partial \Omega^{j}$ and $f^{j}$ denote the germs of $\partial \Omega$ and $f$ at $p_{j}$. We may assume $p_{j} \in \partial \Omega$ and $f^{j}$ has the form (4.2), Remark 1. By a direct calculation, then, we see that if $\left(f^{2}\right)^{-1} f^{1}$ maps $\partial \Omega^{1}$ and $\partial \Omega^{2}$ then $M_{1}=M_{2}$, i.e. the orders are the same.

This theorem has some implications for the construction of proper mappings. Let us recall one of the standard methods of constructing proper maps (see Rudin [81, p. 301]). Let $f: \tilde{\Omega} \rightarrow \tilde{D}$ be a holomorphic mapping such that $f^{-1}(w)$ is compact for $w \in \tilde{D}$. Then for any $p \in \tilde{\Omega}$ there is an arbitrarily small connected neighborhood $\Omega$ of p such that $f: \Omega \rightarrow f(\Omega)$ is proper. The domain $\Omega$ is obtained by taking a connected component of $f^{-1}(D)$ where $D$ is a neighborhood of $f(p)$, which may be chosen with great freedom. In contrast, there is not much freedom in choosing $\Omega$ if $f$ is not locally biholomorphic at $p$. If we wish to choose $\Omega$ to be strongly pseudoconvex, then (if $p \in V_{f}$ ) $\Omega$ must have a nontrivial automorphism group. On the other hand (see $[30,53]$ ), a generic $\Omega$ has no automorphisms.

It is evident that the Factorization Theorem above may be used to reduce the study of the branch locus to the study of $\Gamma_{f}$. Thus far $\Gamma_{f}$ has been identified only in the case $\Omega=\mathbf{B}^{n}$.

A finite subgroup $G \subset U(n)$ of the unitary group is called a reflection group if it is generated by its reflections, i.e. transformations which are conjugate to a diagonal matrix of the form

$$
\left(\begin{array}{ccc}
e^{2 \pi i / k} & & 0 \\
1 & & \\
& \ddots & \\
0 & & 1
\end{array}\right)
$$

It is a classical theorem of Chevalley that the invariant polynomials of a reflection group are generated by $n$ homogeneous polynomials $p_{1}, \ldots, p_{n}$, which we denote by $P_{\Gamma}=\left(p_{1}, \ldots, p_{n}\right)$. Examples of $P_{\Gamma}$ are given in Rudin [83], where the following is proved.

THEOREM. Let $f: \mathbf{B}^{n} \rightarrow D, n \geqslant 2$, be a proper mapping. Then $\Gamma_{f}$ has the following properties:

(i) there exists $T \in \operatorname{Aut}\left(\mathbf{B}^{n}\right)$ such that $0 \in \mathbf{B}^{n}$ is a fixed point of $T^{-1} \Gamma_{f} T$.

(ii) $T^{-1} \Gamma_{f} T \subset U(n)$ is a finite reflection group.

(iii) the mapping $f$ may be factored as $f=\tilde{f} P_{\Gamma} T$, where $\tilde{f}: P_{\Gamma}\left(\mathbf{B}^{n}\right) \rightarrow D$ is a biholomorphism. 
Since the fixed point set of a reflection is a complex linear hyperplane, it follows that $J_{F}=T H_{1} \cup \cdots \cup T H_{k}$, where $H_{j}$ is a complex hyperplane passing through the origin, and $T$ is from the theorem above.

Let us remark that in Rudin's Theorem, the hypothesis that $D$ is a nonsingular complex manifold is crucial. For if $G \subset \operatorname{Aut}\left(B^{n}\right)$ is any finite subgroup, then $\mathbf{B}^{n} / G$ has the structure of a complex space (possibly with singularities), see [31]. Thus for the quotient $f: \mathbf{B}^{n} \rightarrow \mathbf{B}^{n} / G$ we have $\Gamma_{f}=G$, if the image $D=\mathbf{B}^{n} / G$ is allowed to be singular.

6. Mappings into higher-dimensional spaces. Here we present some results concerning proper maps $f: \Omega \rightarrow D$ with $\operatorname{dim} \Omega<\operatorname{dim} D$, but so little is known that we also mention some open problems. Although most of these problems have been known for some time, it seems worthwhile to state them all in the same place.

A. Convex imbedding theorem. The following result of J. E. Fornaess [47] and Henkin [96] is useful, e.g. in approximation results.

THEOREM. If $\Omega \subset \subset \mathbf{C}^{n}$ is strongly pseudoconvex, then there exists a proper imbedding $f: \Omega \rightarrow D \subset \subset \mathbf{C}^{n}$, where $D$ is a strongly convex domain, and $f(\Omega)$ intersects $\partial D$ transversely.

A natural question that arises is: Can $D$ be replaced by the ball $\mathbf{B}^{N}$ ? A result of J. Faran [46] indicates that this is unlikely. Faran's result is: there is a real-analytic strongly pseudoconvex hypersurface $\Gamma \subset \mathbf{C}^{n}$ and a point $p \in \Gamma$ such that there is no holomorphic imbedding of a neighborhood of $p$ in $\Gamma$ into $\partial \mathbf{B}^{N}$ for any $N<\infty$.

On the other hand, L. Lempert [63] has shown that $\Omega$ may be properly imbedded in the unit ball in Hilbert space.

B. Imbedding in $\mathbf{C}^{N}$. It is a basic result of Bishop, Narasimhan, and Remmert $[28,68,77]$ that every $n$-dimensional Stein manifold $M$ can be properly imbedded in $\mathbf{C}^{N}$ for $N=2 n+1$. The problem is to make $N$ as small as possible. By Bishop [28] there is a holomorphic mapping $f: M \rightarrow \mathbf{C}^{n}$ which is "almost proper" (i.e. all connected components of $f^{-1}(K)$ are compact if $K$ is compact). Also by [28] there is a proper mapping $f: M \rightarrow \mathbf{C}^{n+1}$.

Forster [49] has shown that $M$ may be properly imbedded in $C^{N}$ with $N=[5 n / 3]+2$. It was announced in [54] that $M$ may be immersed in $\mathbf{C}^{N}$, properly immersed in $\mathbf{C}^{N+1}$, and properly imbedded in $\mathbf{C}^{N+2}$, with $N=[3 n / 2]$; but the details of the proofs have not yet appeared. For topological reasons, $M$ cannot be imbedded in $\mathbf{C}^{N}, N=[3 n / 2]$. It is an open question whether an imbedding is possible with $N=[3 n / 2]+1$.

A specific case of this question is whether it is possible to imbed an open Riemann surface properly in $\mathbf{C}^{2}$. This imbedding is possible in the cases of the disk, punctured disk, and annulus (see $[4,62]$ ).

C. Boundary regularity of imbeddings. There are proper imbeddings $f$ : $\Delta \rightarrow \mathbf{B}^{2}$ with $f \notin C(\bar{\Delta})$. To give an example, we choose $\phi_{1}, \phi_{2} \in C(\partial \Delta)$ such that $e^{2 \phi_{1}}+e^{2 \phi_{2}}=1$. We may choose $\phi_{1}, \phi_{2}$ such that the harmonic conjugates $\phi_{1}^{*}, \phi_{2}^{*}$ are not continuous, but in any case $f=\left(\exp \left(\phi_{1}+i \phi_{1}^{*}\right), \exp \left(\phi_{2}+i \phi_{2}^{*}\right)\right)$ is proper. 
The problem remains, however, as to whether a proper imbedding (or merely proper mapping) $f: \Omega \rightarrow D$ extends smoothly to $\bar{\Omega}$ if $\Omega, D$ are both smooth and strongly pseudoconvex, and $\operatorname{dim} \Omega \geqslant 2$.

Results in this direction, involving generalizations of the Reflection Principle, have been obtained by Webster [93] and Cima, Krantz and Suffridge [33].

D. Mapping balls to balls. The following was obtained by S. Webster [92].

THEOREM. If $f: \mathbf{B}^{n} \rightarrow \mathbf{B}^{n+1}, n \geqslant 3$, is proper, $f \in C^{3}\left(\overline{\mathbf{B}}^{n}\right)$, and $f$ gives an immersion of $\partial \mathbf{B}^{n}$ into $\partial \mathbf{B}^{n+1}$, then there exists $\psi \in \operatorname{Aut}\left(\mathbf{B}^{n+1}\right)$ such that $\psi f\left(z_{1}, \ldots, z_{n}\right)=\left(z_{1}, \ldots, z_{n}, 0\right)$.

Since the case $\mathbf{B}^{1} \rightarrow \mathbf{B}^{2}$ is impossible, it is perhaps not surprising that $\mathbf{B}^{2} \rightarrow \mathbf{B}^{3}$, the "borderline" case, is more complicated.

THEOREM (FARAN [44]). Let $f: \mathbf{B}^{2} \rightarrow \mathbf{B}^{3}$ be a proper map with $f \in C^{3}\left(\overline{\mathbf{B}}^{2}\right.$ ). Then there are $\psi_{2} \in \operatorname{Aut}\left(\mathbf{B}^{2}\right)$ and $\psi_{3} \in \operatorname{Aut}\left(\mathbf{B}^{3}\right)$ such that $\psi_{3} f \psi_{2}$ is one of the following:

(1) $(z, w) \rightarrow\left(z^{3}, w^{3}, \sqrt{3} z w\right)$,

(2) $(z, w) \rightarrow\left(z, z w, w^{2}\right)$;

(3) $(z, w) \rightarrow\left(z^{2}, \sqrt{2} z w, w^{2}\right)$;

(4) $(z, w) \rightarrow(z, w, 0)$.

The regularity hypothesis $f \in C^{3}\left(\overline{\mathbf{B}}^{n}\right)$ of Webster's Theorem has been weakened in $[34,33]$, but it is not known whether any regularity hypothesis is necessary.

If $f: \mathbf{B}^{n} \rightarrow \mathbf{B}^{k}$ is proper, $k \leqslant 2 n-2$, and if $f$ is holomorphic in a neighborhood of $\overline{\mathbf{B}}^{n}$, then Faran has shown that $f$ is linear fractional (see $[34,45]$ ).

E. Mapping the ball to the polydisk. It is known that there is no proper mapping between polydisk $\Delta^{n}$ and ball $\mathbf{B}^{n}$, e.g., [80]. There are generalizations of this showing that a polyhedron cannot be properly mapped to a domain with strongly pseudoconvex points $[\mathbf{5 7}, \mathbf{5 8}, \mathbf{5 1}]$. Although it is easily seen that $\Delta^{n}$ cannot be mapped properly to $\mathbf{B}^{N}$, for any $N>0$, it is unknown whether it is possible to map $\mathbf{B}^{n}$ properly to $\Delta^{N}$ (of course $N>n$ ).

F. Mappings which are nearly proper. It is of interest to consider mappings $f: \Omega \rightarrow D$ which are close to being proper. For instance, the mapping of the disk $f: \Delta \rightarrow D$, which realizes the Kobyashi metric, is such a map (see E. Poleckii [75]).

Given a mapping $f: \Omega \rightarrow D$, we may consider the exceptional set

$$
E=\left\{z_{0} \in \partial \Omega: \limsup _{z \rightarrow z_{0}} \operatorname{dist}(f(z), \partial D)>0\right\} .
$$

If $E$ is small, is $f$ necessarily proper? This seems not to be known even in the simplest cases, e.g., when $E$ is a point and $\operatorname{dim} \Omega=\operatorname{dim} D \geqslant 2$. (The case $\operatorname{dim} \Omega=1$ is, in general, false.) It is also possible to formulate the same question in terms of the radial exceptional set

$$
E_{\text {rad }}=\left\{z_{0} \in \partial \Omega: \lim \sup _{\varepsilon \rightarrow 0} \operatorname{dist}\left(f\left(z_{0}-\varepsilon N\left(z_{0}\right)\right), \partial D\right)>0\right\},
$$


where $N\left(z_{0}\right)$ denotes the outward normal to $\partial \Omega$ at $z_{0}$. A Baire category argument (cf. Sadullaev [84]) may be given to show that if $E_{\mathrm{rad}}=\varnothing$, then $E \subset \partial \Omega$ is a nowhere dense $F_{\sigma}$-set.

To illustrate this question we consider mappings $f: \Omega \rightarrow \Delta$ with $\operatorname{dim} \Omega \geqslant 2$, which can in no sense be close to proper. The condition that $E_{\text {rad }}$ has zero measure in $\partial \Omega$ is equivalent to $f$ being an "inner" function. Since inner functions have been shown to exist $[2,56,67]$, the condition that $\left|E_{\text {rad }}\right|=0$ is too weak. The other extreme is the case $E_{\mathrm{rad}}=\varnothing$, in which case Sadullaev [84] has shown that such $f$ do not exist.

\section{BIBLIOGRAPHY}

1. L. Ahlfors, Open Riemann surfaces and extremal problems on compact subregions, Comment Math. Helv. 24 (1950), 100-132.

2. A. Aleksandrov, Math. Sb. 118 (160) (1982), 147-163. (Russian)

3. H. Alexander, Proper holomorphic mappings in $\mathbf{C}^{n}$, Indiana Univ. Math. J. 26 (1977), $137-146$.

4. $539-544$.

, Explicit imbedding of the ( punctured) disk into $\mathbf{C}^{2}$, Comment Math. Helv. 52 (1977),

5. D. Barrett, Regularity of the Bergman projection on domains with transverse symmetries, Math. Ann. 258 (1982), 441-446.

6. Boundary analyticity of proper holomorphic maps of domains with non-analytic boundaries, Math. Ann. 263 (1983), 479-482.

7. _ Holomorphic equivalence and proper mapping of bounded Reinhardt domains not containing the origin.

8. _ regular.

9. E. Bedford, Holomorphic mapping of products of annuli in $\mathbf{C}^{n}$, Pacific J. Math. 87 (1980), 27-281.

10. Proper holomorphic mappings from strongly pseudoconvex domains, Duke Math. J. 49 (1982), 477-484.

11. _ Proper holomorphic mappings from domains with real analytic boundary, Amer. J. Math.

12. E. Bedford and S. Bell, Proper self maps of weakly pseudoconvex domains, Math. Ann. 261 (1982), 47-49.

13. Boundary continuity of proper holomorphic correspondences, Séminaire DolbeaultLelong-Skoda, 1983.

14. _ Proper correspondences of bounded domains in $\mathbf{C}^{n}$, Proc. Colloque Analyse Complexe, Toulouse.

15 . , Boundary behavior of proper holomorphic correspondences.

16. E. Bedford, S. Bell and D. Catlin, Boundary behavior of proper holomorphic mappings, Michigan Math. J. 30 (1983), 107-111.

17. E.Bedford and J. E. Fornaess, Biholomorphic maps of weakly pseudoconvex domains, Duke Math. J. 45 (1978), 711-719.

18. S. Bell, Biholomorphic mappings and the $\bar{\partial}-$ problem, Ann. of Math. (2) 114 (1981), 103-113.

19. __ Proper holomorphic mappings and the Bergman projection, Duke Math. J. 48 (1981), $167-175$.

20. Analytic hypoellipticity of the $\bar{\partial}-$ Neumann problem and extendability of holomorphic mappings, Acta Math. 147 (1981), 109-116.

21. , Proper holomorphic mappings between circular domains, Comment Math. Helv. 57 (1982), 532-538.

22. B_ Boundary behavior of proper holomorphic mappings between non-pseudoconvex pseudoconvex domains, Amer. J. Math.

23. $ـ$ Regularity of the Bergman projection in certain non-pseudoconvex domains, Pacific J.

Math. 105 (1983), 273-278. 
24. , Local boundary behavior of proper holomorphic mappings, Proc. Sympos. Pure Math., vol. 40, Amer. Math. Soc., Providence, R. I., 1984. (to appear).

25. S. Bell and H. Boas, Regularity of the Bergman projection in weakly pseudoconvex domains, Math. Ann. 257 (1981), 23-30.

26. S. Bell and D. Catlin, Boundary regularity of proper holomorphic mappings, Duke Math. J. 49 (1982), 385-396.

27. V. K. Beloshapka and A. G. Vitushkin, Estimates for the radius of convergence of power series defining mappings of analytic hypersurfaces, Izv. Akad. Nauk SSSR 45 (1981), 962-984.

28. E. Bishop, Mappings of partially analytic spaces, Amer. J. Math. 83 (1961), 209-242.

29. D. Burns and S. Shnider, Geometry of hypersurfaces and mapping theorems in $\mathbf{C}^{n}$, Comment Math. Helv. 54 (1979), 199-217.

30. D. Burns, S. Shnider and R. O. Wells, Deformation of strongly pseudoconvex domains, Invent Math. 46 (1978), 237-253.

31. H. Cartan, Quotients of complex analytic spaces, Contribution to Function Theory, Oxford Univ. Press, Bombay, 1960.

32. D. Catlin, Boundary invariants of pseudoconvex domains (to appear).

33. J. Cima, S. Krantz and T. Suffridge, A reflection principle for proper holomorphic mappings of strongly pseudoconvex domains and applications.

34. J. Cima and T. Suffridge, $A$ reflection principle with applications to proper holomorphic mappings, Math. Ann.

35. K. Diederich and J. E. Fornaess, Pseudoconvex domains: Bounded strictly plurisubharmonic exhaustion functions, Invent Math. 39 (1977), 129-147.

36. $371-384$.

37. __ Biholomorphic mappings between certain real analytic domains in $\mathbf{C}^{n}$, Math. Ann. 245 (1979), 255-272.

38. __ Proper holomorphic images of strictly pseudoconvex domains, Math. Ann. 259 (1982), 279-286.

39. $363-384$.

40. , Boundary regularity of proper holomorphic mappings, Invent Math. 67 (1982),

Ann. of Math. (2) 110 (1979), 575-592.

41. _ A remark on a paper of $S . R$. Bell, Manuscripta Math. 34 (1981), 31-44.

42. K. Diederich and I. Lieb., Konvexität in der komplexen Analysis, Birkhäuser, 1981.

43. K. Diederich and S. Webster, A reflection principle for degenerate real hypersurfaces, Duke Math. J. 47 (1980), 835-843.

44. J. Faran, Maps from the two-ball to the three-ball, Invent. Math. 68 (1982), 441-475.

45. The linearity of proper holomorphic maps between balls in the low codimension case.

46. The non-imbeddability of real hypersurfaces in spheres (to appear).

47. J. E. Fornaess, Embedding strictly pseudoconvex domains in convex domains, Amer. J. Math. 98 (1976), 529-569.

48. Bilohomorphic mappings between weakly pseudoconvex domains, Pacific J. Math. 74 (1978), 63-65.

49. O. Forster, Plongements des variètés de Stein, Comment Math. Helv 45 (1970), 170-184.

50. B. L. Fridman, On a class of analytic polyhedra, Dokl. Akad. Nauk SSSR $242(1978)=$ Soviet Math. Dokl. 19 (1979), 1258-1961.

51 , On the imbedding of a strictly pseudoconvex domains in a polyhedron, Dokl. Akad. Nauk USSR 249 (1979) = Soviet Math. Dokl. 20 (1979), 1228-1232.

52. _ One example of the boundary behaviour of biholomorphic transformations, Proc. Amer. Math. Soc. 89 (1983), 226-228.

53. R. Greene and S. Krantz, Deformation of complex structures, estimates for the $\bar{\partial}$-equation, and stability of the Bergman kernel, Adv. in Math. 43 (1982), 1-86.

54. M. Gromov and Ja. Eliašberg, Nonsingular mappings of Stein manifolds, Functional Anal. Appl. 5 (1971), 156-157.

55. H. Grunsky, Lectures on theory of functions in multiply connected domains, Vandenhoeck \& Ruprecht, Göttingen, 1978. 
56. M. Hakim and N. Sibony, Fonctions holomorphes bornées sur la boule unité de $\mathrm{C}^{n}$, Invent Math. 67 (1982), 213-222.

57. G. Henkin, An analytic polyhedron is not holomorphically equivalent to a strictly pseudoconvex domains, Dokl. Akad. Nauk SSSR 210 (1973), 1026-1029 = Soviet Math. Dokl. 14 (1973), 858-862.

58. A. Huckleberry and E. Ormsby, Nonexistence of proper holomorphic maps between certain complex manifolds, Manuscripta Math. 26 (1978/79), 371-379.

59. J. Kohn, Subellipticity of the $\bar{\partial}$-Neumann problem on pseudoconvex domains: sufficient conditions, Acta Math. 142 (1979), 79-122.

60. __ Boundary regularity of $\bar{\partial}$, Recent Developments in Several Complex Variables (J. E. Fornaess, ed.), Princeton Univ. Press, Princeton, N. J., 1981.

61. M. Landucci, Proper equivalence for a class of pseudoconvex domains, Trans. Amer. Math. Soc. (to appear).

62. H. Laufer, Imbedding annuli in $\mathrm{C}^{2}$, J. Analyse Math. 26 (1973), 187-215.

63. L. Lempert, Imbedding strictly pseudoconvex domains into a ball, Amer. J. Math. 104 (1982), 901-904.

64. T. C. Li, Proper holomorphic mappings over bounded domains in $\mathbf{C}^{n}$, Bull. Inst. Math. Acad. Sinica 6 (1978), 175-180.

65. __ Proper self-mappings of the Hartogs triangle, Chinese J. Math. 6 (1978), 191-195.

66. E. Ligocka, On proper holomorphic and biholomorphic mappings between product domains, Bull. Acad. Polon. Sci. Sér. Sci. Math. 28 (1980), 319-323.

67. E. Lbw, $A$ construction of inner functions on the unit ball in $\mathbf{C}^{p}$, Invent Math. 67 (1982), 223-229.

68. R. Narasimhan, Imbedding of holomorphically complete complex spaces, Amer. J. Math. 82 (1960), 917-934.

69. Several complex variables, Univ. of Chicago Press, Chicago, 1971.

70. S. Pinčuk, On proper holomorphic mappings of strictly pseudoconvex domains, Siberian Math. J. 15 (1974), 909-917.

71. On the analytic continuation of holomorphic mappings, Mat. Sb. 98(140) (1975), 416-435 = Math. USSR-Sb. 27 (1975).

72. On holomorphic mappings of real analytic hypersurfaces, Mat. Sb. 105(147) $(1978)=$ Math. USSR-Sb. 34 (1978), 503-519.

73. 19 (1978), 804-807.

74. _ Holomorphic inequivalence of some classes of domains in $\mathbf{C}^{n}$, Math. USSR-Sb. 39 $(1981)=$ Mat. Sb. 111(153) (1980), 61-86.

75. E. Poleckii, The Euler-Lagrange equations for extremal holomorphic mappings of the unit disk.

76. M. Range, The Carathéodory metric and holomorphic maps on a class of weakly pseudoconvex domains, Pacific J. Math. 78 (1978), 173-189.

77. R. Remmert, Sur les espaces analytiques holomorphement separables et holomorphement convexes, C. R. Acad. Sci. Paris 243 (1956), 118-121.

78. R. Remmert and K. Stein, Eigentliche holomorphe Abbildungen, Math. Z. 73 (1960), 159-189.

79. H. Rischel, Ein Satz über eigentliche holomorphe Abbildungen von analytischen Polyedergebieten, Math. Scand. 14 (1964), 220-224.

80. __ Holomorphe Überlagerungskorrespondenzen, Math. Scand. 15 (1964), 49-63.

81. W. Rudin, Function theory on the unit ball of $\mathbf{C}^{n}$, Springer-Verlag, 1980.

82. __ Holomorphic maps that extend to automorphisms of a ball, Proc. Amer. Math. Soc. 81 (1981), 429-432.

83. _ Proper holomorphic maps and finite reflection groups, Indiana Univ. Math. J. 31 (1982), 701-720.

84. A. Sadullaev, Inner functions in $\mathbf{C}^{n}$, Math. Notes 19 (1976), 37-38.

85. S. E. Saronov, On holomorphic mappings of polyhedra, Mat. Sb. 116(158) $(1981)=$ Math. USSR-Sb. 44 (1983), 117-123.

86. G. Schüller, Beiträge zur Theorie der randeigentlichen Korrespondenzen, Dissertation, Univ. Duisberg, 1980.

87. R. R. Simha, Über die kritischen Werte gewisser holomorpher Abbildungen, Manuscripta Math. 3 (1970), 97-104. 
88. K. Stein, Maximale holomorphe und meromorphe Abbildungen. II, Amer. J. Math. 86 (1964), 823-868.

89. 443-463.

, Topics on holomorphic correspondences, Rocky Mountain J. Math. 2 (1972),

90. A. Vitushkin, Holomorphic extension of mappings of compact hypersurfaces, Math. USSR-Izv. 20 (1983), 27-33 = Izv. Akad. Nauk SSSR 46 (1982).

91. S. Webster, On the mapping problem for algebraic real hypersurfaces, Invent Math. 43 (1977), 53-68.

92. On mapping an $n$-ball into an $(n+1)$-ball in complex space, Pacific J. Math. 81 (1979), 267-272.

93. Holomorphic mappings of domains with generic corners, Proc. Amer. Math. Soc. 86 (1982), 236-240.

94. B. Wong, A maximum principle on Clifford torus and nonexistence of proper holomorphic map from the ball to polydisk, Pacific J. Math. 87 (1980), 211-222.

95. J. P. D’Angelo, Real hypersurfaces, orders of contact, and applications, Ann. of Math. (2) 115 (1982), 615-637.

96. G. Henkin and E. Chirka, Boundary properties of holomorphic functions of several complex variables, J. Soviet Math. 5 (1976), 612-687.

97. S. Pincuk, On the boundary behavior of analytic sets and algebroid mappings, Dokl. Akad. Nauk USSR 268 (1983), 296-298. (Russian)

DePaRTMENT OF MATHEMATICS, INDIANA UNiverSity, BlOOMINGTON, INDIANA 47401 
